CORRECTION

D) Check for updates

\title{
Correction: Targeting the HIF-1a-IGFBP2 axis therapeutically reduces IGF1-AKT signaling and blocks the growth and metastasis of relapsed anaplastic Wilms tumor
}

Yan Liu (D), Marie V. Nelson, Christopher Bailey, Peng Zhang, Pan Zheng, Jeffrey S. Dome, Yang Liu (D) and Yin Wang (iD

(c) The Author(s), under exclusive licence to Springer Nature Limited 2021

Oncogene (2022) 41:1383; https://doi.org/10.1038/s41388-021-02042-7

Correction to: Oncogene (2021) 40:4809-4819, https://doi.org/ 10.1038/s41388-021-01907-1, published online 21 June 2021
Following the publication of this article, it was noted that co-first authorship was omitted. The article has been updated to include Yan Liu and Marie V. Nelson as co-first authors.

The original article has been corrected. 